\title{
Atomic Resolution Probing of Phase Transformations and Domain Evolution During Large Superelastic Deformation in Ferroelectrics with in situ TEM
}

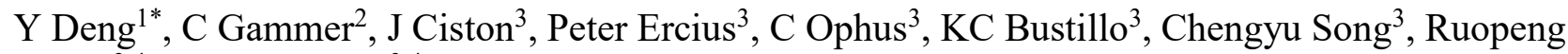 \\ Zhang ${ }^{3,4}$ and AM Minor ${ }^{3,4}$ \\ 1. Physics School and Modern Analysis Center, Nanjing University, Nanjing, China. \\ 2. Erich Schmid Institute of Materials Science, Austrian Academy of Sciences, Leoben, Austria. \\ 3. National Center for Electron Microscopy, Molecular Foundry, Lawrence Berkeley National \\ Laboratory, Berkeley, CA, USA. \\ 4. Department of Materials Science \& Engineering, University of California, Berkeley, CA, USA. \\ * Corresponding author: dengyu@nju.edu.cn
}

Intrinsically brittle Ferroelectrics have attractive applications such as flexible/wearable electronic devices and mechanically-written high-density memory that depend on both functional and mechanical performance [1-3]. One potential way to mitigate their brittle behavior is through large superelastic deformation stemming from strain-driven phase transformations and domain evolution. Therefore, we investigated free-standing single-crystal $\mathrm{BaTiO}_{3}$ and $\mathrm{Pb}\left(\mathrm{Mg}_{1 / 3} \mathrm{Nb}_{2 / 3}\right) \mathrm{O}_{3}-\mathrm{PbTiO}_{3}$ sub-micrometer pillars during large superelastic deformation, directly observing the stress-induced phase transformations and domain evolution by using in situ Scanning Transmission Electron Microscope (STEM). We found ultra-small and multiple-phase coexistence with sizes down to a few unit cells, and we were able to study them at atomic resolution while under load. Notably, extremely small phase transformation structures and domain structures are often introduced during the large superelastic deformations since the stress field is not screened in ferroelectrics, as compared to the situation with electrical field or thermal cycling.

We prepared the free-standing sub-micrometer pillars from bulk single crystals of $\mathrm{BaTiO}_{3}$ by Focused Ion Beam (FIB). The in situ observations were carried out using a probe and imaging Cs-corrected (S)TEM from FEI providing sub-atomic resolution (TEAM I at NCEM) equipped with a direct-electrondetector (K2-IS, Gatan). In situ mechanical testing was carried out using a picoindenter TEM holder (PI95, Hysitron). To limit the influence of the electron beam, we performed TEM observations using a low current density $\left(\leq 10^{-2} \mathrm{~A} / \mathrm{cm}^{2}\right)$ and STEM measurements were performed using a relatively low beam current $(\leq 35 \mathrm{pA})$. During deformation we performed four-dimensional scanning transmission electron microscope (4D-STEM) to provide phase and strain information from the unit-cell to micrometer scale in order to map the deformed microstructure while the samples were under load [4-5].

Through our in situ S/TEM experiments we were able to quantitatively study the nucleation, forward growth, side growth and merging of the twinning domains and bundle domains (as shown in Figure 1). At atomic resolution, we were able to observe the nucleation and growth of the strain-driven phase transformation structures during the large superelastic deformation. The distributions of local strains and mobile point defects were also analyzed. Based on our experimental findings, the 'controversial' large superelastic deformation in 'brittle' ferroelectrics can be well understood with a model that accounts for multiple mechanisms contributing at multiple length scales.

In conclusion, the full superelastic deformation process and the strain-driven phase transformation and domain evolution during large superelastic deformation in ferroelectrics has been investigated at 
multiple length scales by using in situ S/TEM. Such observations provide crucial insight into the multiple mechanisms involved in superelastic deformation [6].

\section{References:}

[1] G Catalan et al., Rev. Modern Phys. 84 (2012), p. 119.

[2] JF Scott, Science 315 (2007), p. 954.

[3] YL Tang et al., Science 348 (2015), p. 547.

[4] C Gammer et al., Ultramicroscopy 1 (2015), p. 155.

[5] VB Ozdol et al., Applied Physics Letters 106 (2015), p. 253107.

[6] The authors acknowledge support by the National Natural Science Foundation of China (Grants Nos. 50802039) and Natural Science Foundation of Jiangsu Province, China (Grant Nos. BK20151382), and the National Science Foundation through the STROBE Science and Technology Center. RZ acknowledges funding from the US Office of Naval Research under Grant No. N00014-12-1-0413 and N00014-17-1-2283. The electron microscopy work was performed at the Molecular Foundry, Lawrence Berkeley National Laboratory, which is supported by the Office of Science, Office of Basic Energy Sciences, of the U.S. Dept. of Energy under Contract \# DE-AC02-05CH11231. J.C. acknowledges additional support from the Department of Energy Early Career Research Program.

(a)

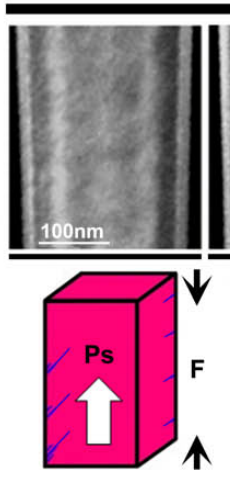

Loading up

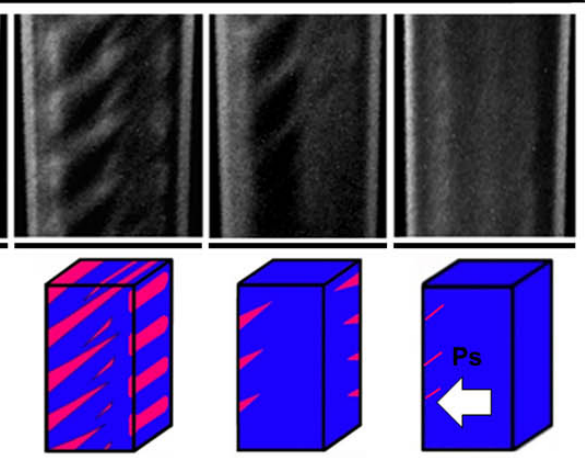

(b)

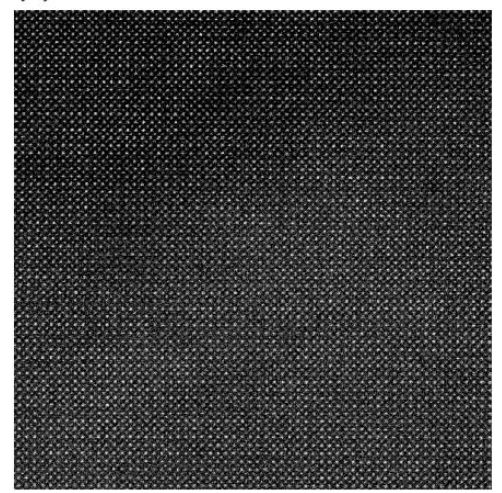

Figure 1. (a) The domain evolutions in a single-crystal $\mathrm{BaTiO}_{3}$ sub-micrometer pillar under compression loading. We use the models below to illustrate the domain evolutions in the pillar. (b) HRSTEM observation on a domain wall. 